\title{
Glucocorticoids act on glutamatergic pathways to affect memory processes
}

\author{
Carmen Sandi
}

Laboratory of Behavioral Genetics, Brain Mind Institute, École Polytechnique Fédérale de Lausanne (EPFL), Lausanne, Switzerland

\begin{abstract}
Glucocorticoids can acutely affect memory processes, with both facilitating and impairing effects having been described. Recent work has revealed that glucocorticoids may affect learning and memory processes by interacting with glutamatergic mechanisms. In this opinion article I describe different glutamatergic pathways that glucocorticoids can affect to modulate memory processes. Furthermore, glucocorticoid-glutamatergic interactions during information processing are proposed as a potential model to explain many of the diverse actions of glucocorticoids on cognition. The model suggests that direct modulation of glutamatergic pathways by glucocorticoids could serve as an important mechanism for these hormones to directly alter cognitive functions.
\end{abstract}

\section{Introduction}

Intensive research in the last decades has uncovered stress as a major regulator of cognitive function. Glucocorticoids are steroid hormones produced by the adrenal glands whose secretion increases under stress [1]. Owing to their lipophilic nature, glucocorticoids can cross the blood-brain barrier to access to the brain where, through binding to specific receptors [mineralocorticoid (MR) and glucocorticoid (GR) receptors] and by means of slow genomic and rapid non-genomic actions, they can have multiple effects on neural function and cognition (Box 1).

Acute and chronic actions of glucocorticoids on memory processes differ in many respects, including differences in behavioral outcomes as well as in the cellular and molecular mechanisms involved. This opinion article focuses on the acute actions of glucocorticoids on memory processes including learning, consolidation, retrieval and extinction (Glossary); [2,3] for reviews on chronic effects). A key feature of the acute effects of glucocorticoids on memory function is that their effects can be quite divergent, with both facilitating and impairing effects [4,5]. Several influential models have accommodated such contradictory findings by classifying effects according to the characteristics of the glucocorticoid response and/or the memory process under study [4-12] (Box 2).

The question arises as to whether a mechanistic explanation can be provided to explain how glucocorticoids produce such a diversity of actions. Given that until recently glucocorticoids were thought to act exclusively via genomic mechanisms, research has focused predominantly

Corresponding author: Sandi, C. (carmen.sandi@epfl.ch). on changes in gene and protein expression in response to glucocorticoids [13-16]. Because genomic mechanisms take some time to develop, such a mechanism cannot apply to extremely rapid effects of glucocorticoids reported for some cognitive operations (for example, learning and retrieval when tests are given shortly after the enhancement of glucocorticoid levels). Importantly, recent work has underscored the potential of glucocorticoids to affect memory processes and synaptic plasticity by interacting with glutamatergic mechanisms (Box 3) through both nongenomic and genomic pathways.

The first part of this article discusses studies that demonstrate glucocorticoid actions on specific aspects of glutamatergic pathways in the context of information processing. These actions include (i) genomic and non-genomic increases in extracellular glutamate levels that affect excitatory transmission, (ii) the activation of NMDA-type

\footnotetext{
Glossary

Consolidation: the process of storage of acquired information.

Extinction: a process that inhibits expression of former learned responses.

Fear conditioning task: a task in which animals learn, by association, that discrete or contextual cues predict aversive conditions.

Forced swim test: a test in which animals placed in an enclosed cylinder full of water learn that there is no escape and eventually develop a floating response. Learning: the process involved in the acquisition of information.

Object location test: a task in which memory for a particular location of two objects to which rodent is exposed in a first phase is indicated by higher levels of exploration of one of the objects that is displaced at testing.

Object recognition test: a task in which rodent recognition of a familiar object is indicated by higher levels of exploration of a novel object when both objects are presented in a free choice test.

Inhibitory or passive avoidance task: a task in which animals learn to inhibit an innate response to avoid receiving an aversive stimulation (such as a footshock).

Priming: a process whereby learning circuits activated by a particular stimulation show reduced threshold for subsequent reactivation by similar stimulation in the near future.

Retrieval: the process of recall of stored information.

Spatial learning: a learning process whereby individuals learn to orientate themselves in their spatial environment by taking into account the location of distal visual cues. The water maze is a common behavioral paradigm used to assess spatial learning in rodents.

Swim-stress paradigm: a stress-induction procedure in which animals are exposed for a defined period of time (that normally varies from 2 to $15 \mathrm{~min}$ ) to a water tank where there is no possibility of escape.

T-maze delayed alternation task: a working memory task in which animals learn to find rewards at the end of two arms in a T-maze by visiting the arm opposite to the previously visited one after being submitted to a certain delay.

Water maze: a behavioral task used to study spatial learning and memory. This task typically consists of a circular water tank in which rodents have to learn to locate a hidden submerged platform using distal visual cues to orientate themselves and navigate in their spatial environment.

Working memory: a cognitive process consisting of keeping recently acquired information 'online' and available to further cognitive operations during a brief period (from seconds to minutes).
} 


\section{Box 1. Glucocorticoids, their receptors and mechanisms of action}

Glucocorticoids (referred to as cortisol in humans and corticosterone in rodents) are the final products of the activated hypothalamus-pituitary-adrenal (HPA) axis. Corticosterone binds to two types of receptors, the mineralocorticoid receptor (MR) and glucocorticoid receptor (GR). Classically, both MR and GR have been identified as intracellular receptors acting as ligand-activated nuclear regulators and exerting slow-onset genomic effects through transrepression and transactivation. Intracellular MRs have a 10-fold higher affinity for corticosterone than GRs, implying that MRs are largely occupied under basal corticosterone conditions, whereas GR occupancy is increased when corticosterone levels rise.
Recently, evidence has emerged for rapid, non-genomic and transient effects of these receptors when expressed at the cell membrane in different brain areas [1] (Figure I). In the hippocampus, lower-affinity membrane-associated MRs were reported to be located presynaptically and to rapidly increase glutamate release probability upon activation [93]. In the lateral amygdala, non-nuclear-membrane GRs were demonstrated to be localized postsynaptically [17]. Membrane-bound GRs that are coupled to G-protein-coupled receptors (GPCRs) have been implicated in the rapid effects of corticosterone in feedback inhibitory actions in the hypothalamus that involve endocannabinoid signaling [25] and in the fast-inducing actions of corticosterone in medial PFC-dependent cognition [88].

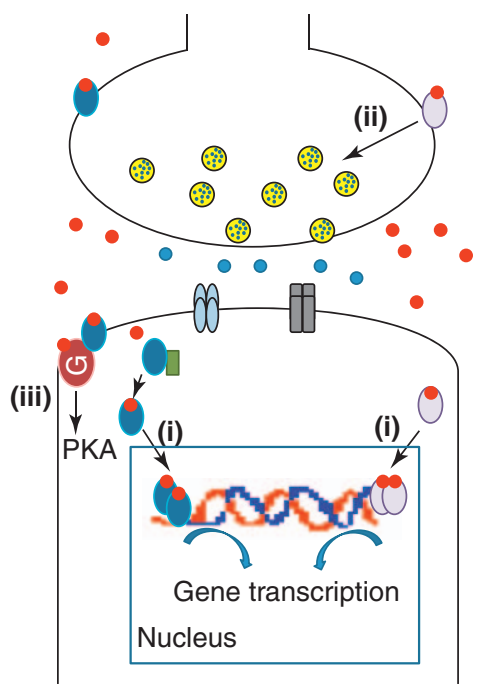

\begin{tabular}{|l|}
\hline Key: \\
- Corticosterone \\
GR \\
GR Stimulatory G protein-coupled receptor \\
(1) Glutamatergic vesicle \\
- Glutamate \\
AMPAR \\
甲 NMDAR \\
\hline
\end{tabular}

$\overline{\text { TRENDS in Neurosciences }}$

Figure I. Schematic representation of the neuronal actions of MR and GR. (i) Upon corticosterone binding, GRs and MRs dissociate from cytoplasmic heat shock proteins. The receptors then translocate to the nucleus where they act as ligand-activated nuclear regulators, affecting gene transcription for a large number of proteins. (ii) Membrane-bound MRs have been described presynaptically and have been shown to increase glutamate release. (iii) Membrane-bound GRs have been shown to be linked to the activation of membrane-associated GPCRs, and this results in the subsequent enhancement of cAMP signaling pathways leading to an increase in protein kinase A (PKA) activity [25].

glutamate receptors (NMDARs) and downstream signaling pathways, and (iii) increased membrane trafficking of AMPA-type glutamate receptors (AMPARs). The latter part of the article presents a model that highlights glucocorticoid-glutamatergic interactions during information processing as a key cellular mechanism that could explain many of the diverse cognitive actions of glucocorticoids.

\section{Glucocorticoid actions on specific aspects of glutamatergic pathways}

Glucocorticoids increase extracellular glutamate levels and affect excitatory transmission

One mechanism whereby glucocorticoids can affect glutamatergic pathways is by increasing extracellular glutamate levels, as described for both stress and elevated glucocorticoids in different brain areas [17]. A rise in peripheral corticosterone levels produces a rapid increase in corticosterone levels in the hippocampus in parallel with a specific increase in extracellular glutamate levels [18]. Glucocorticoid-induced increases in extracellular glutamate levels in the hippocampus can be exerted through a variety of mechanisms, including GR-mediated inhibition of glutamate uptake $[19,20]$ and non-genomic membrane MR- or GR-mediated increase of presynaptic glutamate release probability [21-24]; nevertheless it should also be noted that a GR-mediated decrease in glutamate release probability has been observed in some brain regions such the hypothalamus [25]. However, for this mechanism to be effective it should ideally be capable of affecting excitatory transmission immediately (Figure 1a,b). This has been found to be the case in the hippocampus in connection with the primary actions of corticosterone on increased presynaptic glutamate release. The evidence includes a rapid and reversible enhancement of the frequency of miniature excitatory postsynaptic currents (mEPSCs, currents that exclusively involve AMPARs) in the hippocampal CA1 area [21] and indications of an enhanced likelihood of generating action potentials postsynaptically [1]. More recently, glucocorticoids were also found to enhance glutamatergic transmission rapidly in basolateral amygdala neurons through an MRdependent mechanism [26]. Interestingly, in contrast to the transient effect observed in the CA1 area [21], the enhanced mEPSC activity in the amygdala is long lasting (i.e. maintained for several hours) [26], an effect that requires both rapidly induced MR-dependent [26] as well as delayed GR-dependent $[27,28]$ enhancement of glutamatergic transmission. 


\section{Box 2. Glucocorticoids and their diverse effects on synaptic plasticity and memory processes}

Acute stress can affect synaptic plasticity and cognition by acting in different brain regions. In the hippocampus, stress was systematically shown to impair long-term potentiation (LTP) while facilitating longterm depression (LTD) [94]. Glucocorticoid actions via GR and NMDARs have been implicated in these effects [37,69]. However, acute effects of glucocorticoids on LTP are not always detrimental. In fact, for both LTP and learning and memory, the literature is somewhat confusing, with multiple examples of facilitating and impairing effects. Several models have been put forward to explain these paradoxical findings:

(i) The dose-dependent inverted-U shape model

Effects are explained by corticosterone dosage, with very low and very high corticosterone levels impairing, while intermediate levels facilitate LTP $[10,94]$ and learning (Figure I), particularly in hippocampus-dependent learning tasks $[5,9,11,95]$. Recently, an inverted-U response pattern to increasing corticosterone doses was also demonstrated for PFC-dependent working memory $[57,88]$. However biphasic effects of glucocorticoids are not found in all types of learning [5].

(ii) The convergence in time and space model

Differential effects have been proposed to be related to the timing of when stress or glucocorticoid elevations occur with regards to information processing. Facilitating effects are typically observed when the stress/glucocorticoid peak elevation occurs at around the time of synaptic activity or learning, provided that they affect the same brain areas (i.e. 'space') activated by the particular learning experience [4]. The relevance of the 'convergence' of these factors

Although the implications of these rapid glucocorticoid effects on glutamatergic transmission for memory processing have not yet been elucidated, rapid behavioral changes have been reported after corticosterone injections [29] with indirect evidence for the potential ivolvement of glutamatergic pathways [30].

\section{Glucocorticoids impair memory retrieval by activating extrasynaptic GluN2B-containing NMDA receptors}

Pioneering studies have shown that stress and glucocorticoids given $30 \mathrm{~min}$ (but not $2 \mathrm{~min}$ or $4 \mathrm{~h}$ ) before the performance of recall tasks impair memory retrieval for spatial information acquired (immediately or $24 \mathrm{~h}$ ) before stress or glucocorticoid administration [31-33]. Subsequent studies have demonstrated that the mechanisms mediating these stress and glucocorticoid effects are the same as those that underlie the induction of long-term depression (LTD) in the hippocampus [34], including the activation of extrasynaptic GluN2B (but not GluN2A)-containing NMDARs and the endocytosis of GluA2-containing AMPARs [35,36]. Specifically, it is thought that activation of hippocampal GRs by corticosterone leads to the blockade of glutamate uptake [20] which, consequently, leads to spillover of synaptically released glutamate by low-frequency stimulation, which then acts on extrasynaptic GluN2B-containing NMDARs, leading to the subsequent endocytosis of GluA2-containing AMPARs (Figure 1c). Such a proposed mechanism is in agreement with evidence that (i) GR activation selectively hampers NMDAR-dependent synaptic plasticity [37], and (ii) blocking glutamate uptake facilitates LTD by allowing glutamate release by low-frequency stimulation to activate extrasynaptic NMDARs [36]. GluN2B-containing NMDARs have also been implicated in mediating the with the 'context' of the learning experience has also been emphasized $[5,7]$.

(iii) The relevance of the memory phase

Typically, glucocorticoids facilitate consolidation but impair retrieval (and working memory) are impairing $[8,12]$. The glucocorticoidinduced retrieval impairment has been linked to the facilitating effects of these hormones on extinction processes $[12,70,85,86]$.

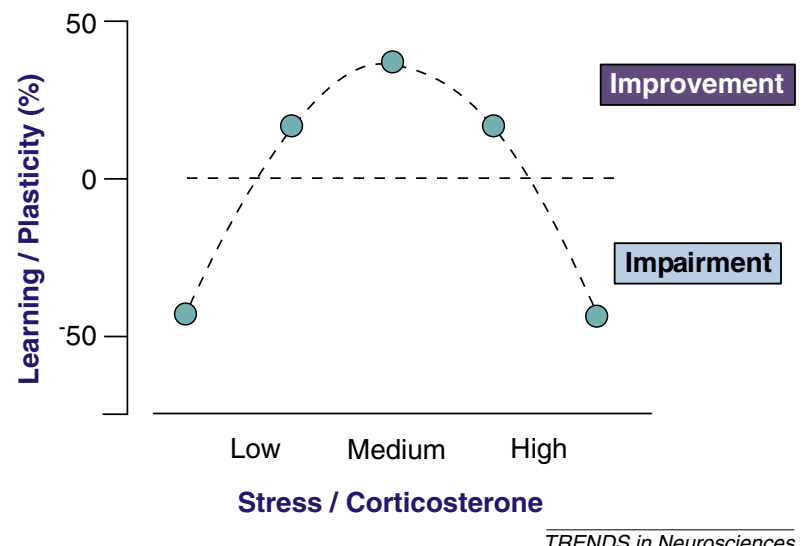

Figure I. Schematic representation of the inverted-U effects of different levels of acute stress or glucocorticoid levels on hippocampus-dependent learning and synaptic plasticity.

impairing effects of stress during memory retrieval as assessed in rats during an object recognition memory task [38].

\section{Glucocorticoids facilitate learning and memory by inducing the synaptic delivery of AMPARs}

Studies performed in rat hippocampal cultured neurons have demonstrated that corticosterone rapidly increases the surface delivery of GluA1- and 2-containing AMPARs, but not GluN1-containing NMDARs [39]. This effect was shown to be non-genomic and dependent upon the activation of membrane MRs. Furthermore, such changes in glutamate receptor levels were demonstrated to have an impact upon the plasticity of the circuit, as revealed by a rapid amplification of the increase of synaptic surface GluA2 content induced by a chemical long-term potentiation (LTP) stimulus [39]. In addition, corticosterone also increased the surface expression of GluA2 AMPAR subunits (and to a lesser extent GluA1, but not GluN1 subunits) in a genomic, GR-dependent, delayed fashion [39]. However, at this later time-point (2-3 h from administration), corticosterone blocked the increase in synaptic GluA2-containing AMPARs elicited by chemical LTP stimulation [39] and facilitated LTD induction [40]. The implication of these findings has been recently postulated within a framework in which stress-elicited rapid, MR-dependent glucocorticoid actions on synaptic transmission and synaptic plasticity in the hippocampus would facilitate the encoding of stress-related information and memories [41]. In addition, the slower, GR-mediated actions triggered by the initial stressful experience would exert a second-wave of insertion of GluA2-containing AMPARs at synaptic sites that would serve to increase synaptic strength and 
promote memory consolidation [41]. Importantly, this model also implies that the GR-mediated increase in the synaptic incorporation of GluA2-containing AMPARs would enhance the threshold for the ability of novel inputs/information, reaching the network hours after the initial stressful learning experience, to be encoded and stored as a new memory [41].

Increased hippocampal AMPAR trafficking has recently been implicated in the facilitating action of corticosterone during spatial learning in mice [42]. This study was based on earlier observations in rats that the strength of water maze spatial memory varies according to the water temperature and the corresponding extent of corticosterone activation $[43,44]$. Mice trained in water at $22{ }^{\circ} \mathrm{C}$ had higher plasma corticosterone levels and better learning and memory than mice trained in water at $30{ }^{\circ} \mathrm{C}$. Immediately after training, only mice trained at $22{ }^{\circ} \mathrm{C}$ showed enhanced synaptic expression of GluA2 (and GluA3, but not of GluA1 or GluN1) subunits. The enhancement of AMPAR subunit GluA2 trafficking was required for the facilitation of memory and was shown to be dependent upon corticosterone action (Figure 1d). By 45 min after training, all GluA1-3 subunits were synaptically enhanced in $22{ }^{\circ} \mathrm{C}$-trained mice (and GluA2 elevations were still observed at $24 \mathrm{~h}$ post-training). In parallel, there was a similar stressful-learning-induced synaptic increase in $\mathrm{N}$ cadherin (a cell-adhesion molecule that interacts with GluA2 subunits and which plays an important role in the formation and growth of dendritic spines [45]). Although this result is purely correlative, it suggests a potential interaction between these two molecules as a mechanism whereby stress can improve memory function (but note that other synaptic cell-adhesion molecules that are also enhanced after stressful learning paradigms could also be involved [46-48]).

An involvement of AMPARs in memory facilitation by corticosterone has also been reported in chicks. In the passive avoidance task, long-term memory involves a training-induced increase in corticosterone levels acting through GRs [49,50]. In a weak training version, intrace- rebral corticosterone administration around the time of training facilitates the transfer of information into longterm memory [51]. Pharmacological experiments showed that whereas both NMDA and AMPA receptors are required at around the time of training, only AMPARs were required during consolidation (i.e. $5.5 \mathrm{~h}$ after training) to mediate the potentiating effects of corticosterone [51].

Moreover, indirect evidence implicates the importance of AMPARs for the formation of fear memories in rodents. In the inhibitory avoidance task (for which long-term memory requires the hippocampus that also mediates GR-induced facilitation on consolidation [52]) NMDARdependent increase in the phosphorylation and synaptic expression of GluA1 and GluA2 subunits, but not GluN1 subunits, was shown [53]. Training in this task increased the amplitude of evoked synaptic transmission in the CA1 region of the hippocampus, similar to that observed during LTP induction. In the fear conditioning task (for which glucocorticoid involvement in consolidation was shown in the amygdala [54] and hippocampus [14,54]), tone conditioning rapidly increases synaptic GluA1 subunit levels in the lateral amygdala, a mechanism that determines the strength of the memory formed [55]. In the hippocampus, this task enhanced AMPAR-mediated synaptic modifications $3 \mathrm{~h}$ post-training [56].

A study also implicated AMPARs in the facilitating effects of stress and glucocorticoids on working memory [57]. Working memory - as tested in a PFC-dependent Tmaze delayed alternation task - was improved, in a GRdependent manner, when rats were tested at $4 \mathrm{~h}$ or $24 \mathrm{~h}$, but not at $48 \mathrm{~h}$ after forced-swim stress. Stress and corticosterone increased postsynaptic glutamatergic transmission via GRs - most likely through the observed postsynaptic delivery of AMPARs and NMDARs - in the PFC ([57]; but note that a negative correlation was recently found between hippocampal GluA2 expression and short-term memory [58]). However, in contrast to the examples discussed previously in this section, the stress/glucocorticoids whose cognitive effects were explored in this latter working-memory study [58] are not those elicited by training, but were instead

\section{Box 3. Glutamate receptors, synaptic plasticity, and memory}

Glutamate is the major excitatory neurotransmitter in the mammalian brain. Upon its release into the synaptic cleft, it can follow different pathways: (i) activating pre- or postsynaptic glutamate receptors, (ii) being recaptured by a glutamate transporter, and transported either back to the presynaptic terminal or into astrocytes, (iii) spilling over from the synaptic cleft, leading to the activation of extrasynaptic glutamate receptors.

There are three main categories of ionotropic glutamate receptors: AMPARs, NMDARs and kainate receptors. AMPA and NMDA receptors are the two main classes that have been studied with respect to glucocorticoid actions; kainate receptors are therefore not discussed further here.

AMPARs are heterotetramers comprised of a combinatorial assembly of four subunits, GluA1-A4 (previously known as GluR1-R4 [96]). AMPARs underlie activity-dependent changes in excitatory synaptic function during synaptic plasticity events and learning. LTP and LTD require the synaptic insertion or removal, respectively, of AMPARs [95], and AMPAR synaptic trafficking has been shown to play an important role in memory formation $[97,98]$.

NMDARs are heterotetrameric channels composed of two obligatory GluN1 (formerly named NR1 [96]) subunits and two regulatory subunits (GluN2A-D or GluN3A,B; formerly NR2A-D or NR3A,B). Most forms of LTP, LTD and memory processes require NMDAR activation and the subsequent cascade of events triggered by $\mathrm{Ca}^{2+}$ influx [99]. NMDARs are localized at both synaptic and extrasynaptic sites. Although still controversial, receptors containing the GluN2A subunit are more likely to be placed centrally in the synapse, whereas those containing the GluN2B subunit are likely to be targeted to peri-synaptic and extrasynaptic sites [100] (Figure I). GluN2A- and GluN2B-containing receptors are thought to be coupled to distinct intracellular signaling pathways, with evidence suggesting their participation in different types of plasticity (LTP and LTD, respectively). The classical view is that memory consolidation involves the activity-dependent-activation of molecular signaling cascades that are initiated by the presynaptic release of glutamate and subsequent activation of AMPA and NMDA receptors, followed by the activation of a variety of kinases (e.g. CaMKII, PKC, PKA, ERK/ MAPK) and downstream signaling pathways, in addition to the induction of new protein synthesis and structural modifications at relevant synapses - such as changes in spine shape or the formation of new dendritic spines [64]. 
(a)

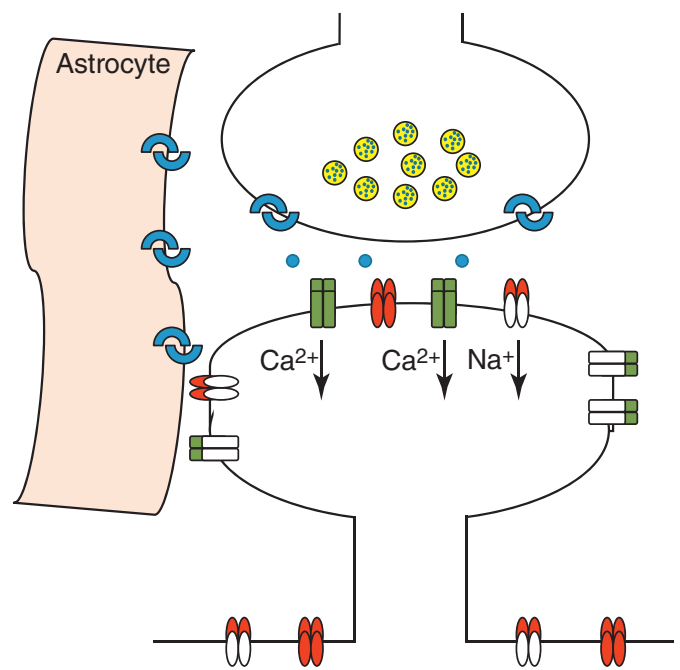

(c)

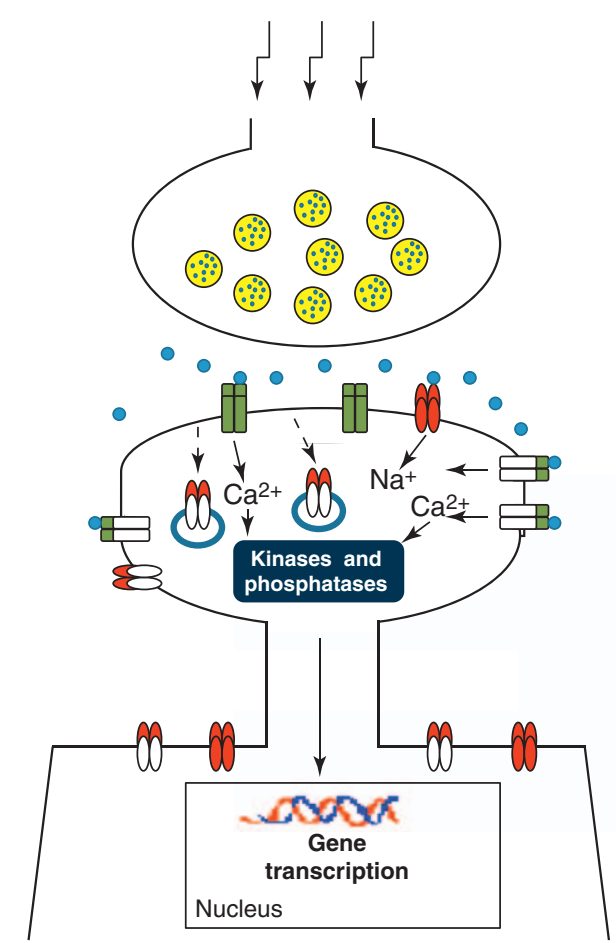

c)

Induction of LTD (b)

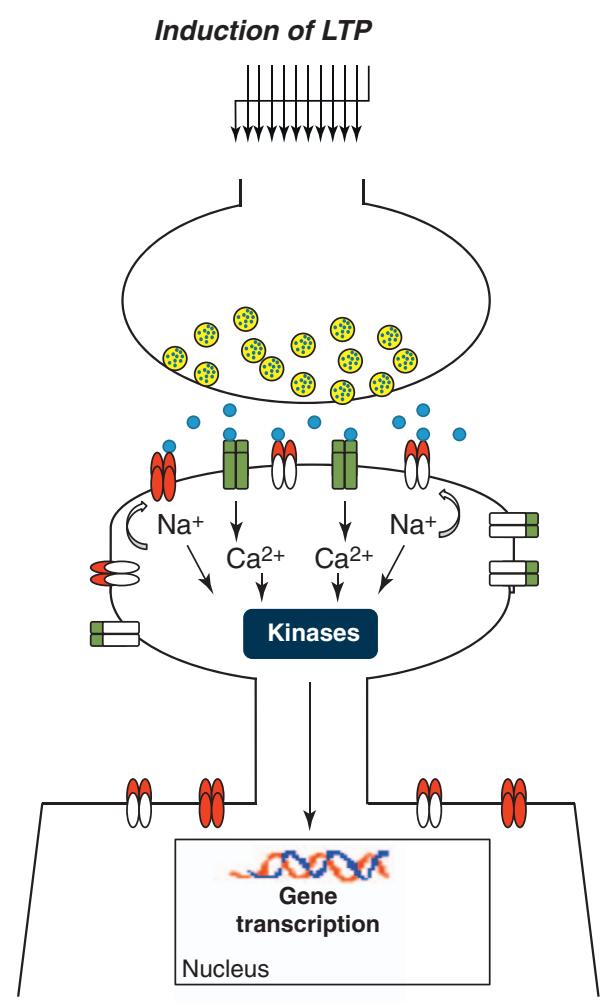

Key:

(7) Glutamatergic vesicle

- Glutamate

GluA1-containing AMPAR

W GluA2-containing AMPAR

用 GluN2A-containing NMDAR

GluN2B-containing NMDAR

Neural transmission

Glutamate transporter

TRENDS in Neurosciences

Figure I. Schematic representation of a glutamatergic synapse under basal conditions and following the induction of synaptic plasticity. Reference to glutamatergic receptors is restricted to AMPAR and NMDAR subtypes. (a) Elements of the glutamatergic synapse under basal conditions. (b) Basic mechanisms involved in the induction of LTP following high-frequency stimulation. Activation of AMPARs by glutamate results in the activation of NMDARs and subsequent influx of Ca ${ }^{2+}$. This influx of $\mathrm{Ca}^{2+}$, together with the activation of signaling pathways that are downstream of the receptors, activate a biochemical cascade involving the activation of various kinases such as CamKII and MAPK/ERK. These pathways ultimately transmit the signals to the nucleus, where changes in gene transcription and translation occur that eventually enhance synaptic strength. The insertion of newly formed AMPARs at synaptic sites, as well as the formation of new spines, is known to occur after the induction of LTP. (c) Basic mechanisms involved in the induction of LTD following low-frequency stimulation. Different mechanisms have been implicated in LTD induction; this diagram represents mechanisms involving the GluN2B subunit of the NMDAR. Glutamate activation of extracellular GluN2B-containing receptors induces $\mathrm{Ca}^{2+}$ influx and GluA2-containing AMPAR endocytosis. Subsequent activation of kinases and phosphatases eventually leads to the induction of gene transcription, the net result being a reduction in synaptic efficacy. 


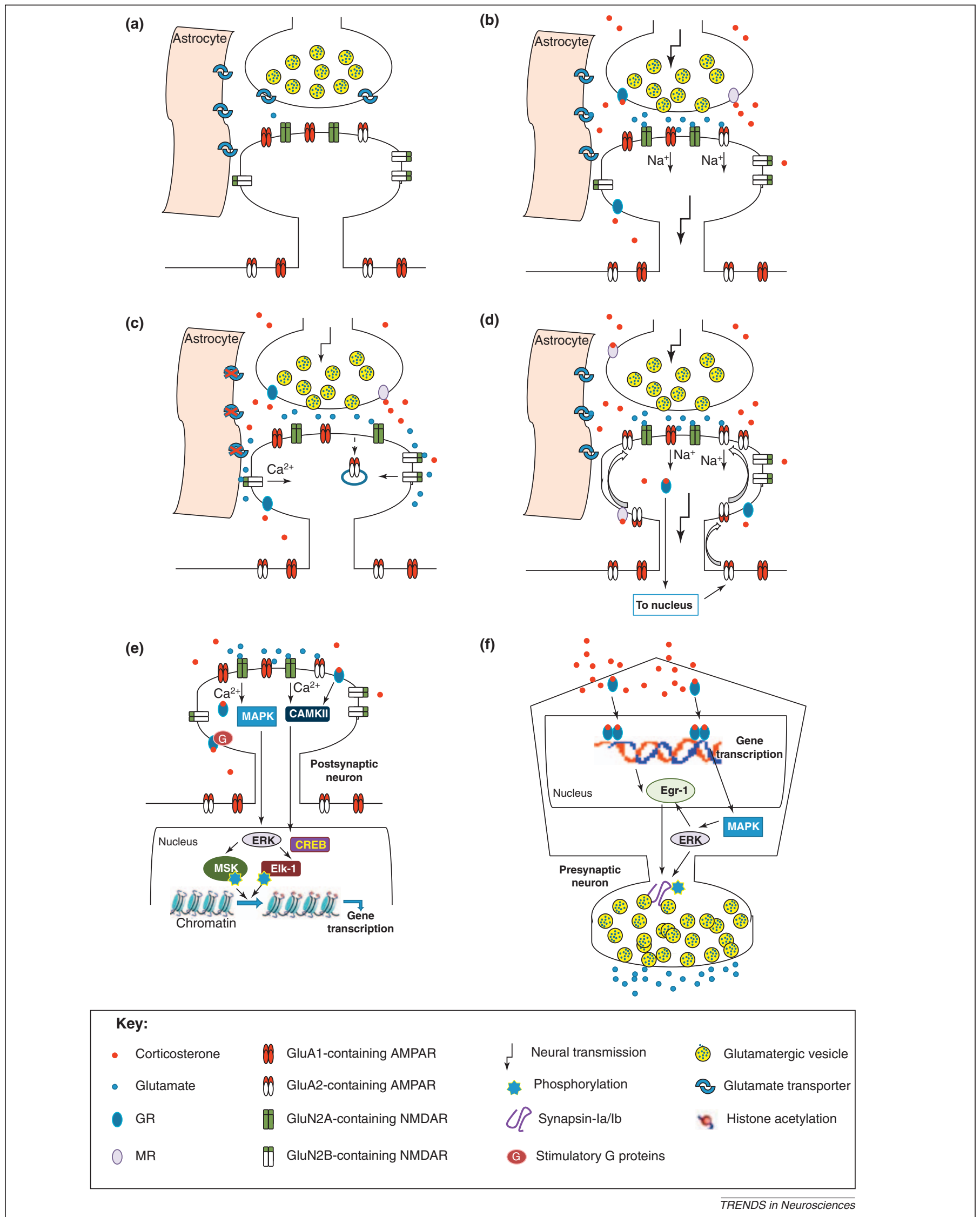

Figure 1. Schematic representations of the different glutamatergic pathways that glucocorticoids can affect to modulate memory processes. (a) Basal conditions. A glutamatergic synapse under basal conditions, including the different key elements of this tripartite unit - the presynaptic neuron (with glutamatergic vesicles and glutamate transporters), the postsynaptic neuron (with a diversity of glutamate receptors located both synaptically and extra-synaptically) and nearby astrocytes (with glial

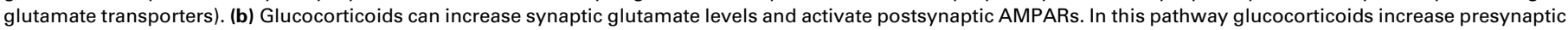
release probability through non-genomic MRs (or GRs), which results in an increase in the amount of glutamate released when the pathway is concurrently activated during 
administered before exposure to the cognitive task. Another important peculiarity of this study is that the facilitating cognitive actions of glucocorticoids were observed in memory tests delivered several hours after treatment [58]. In fact, substantial evidence indicates that when stress or glucocorticoid elevations occur either shortly before or during working memory performance, an impairment is observed $[12,59,60]$.

The cellular mechanisms whereby glucocorticoids result in the enhanced delivery of AMPARs to synapses are not yet well understood; the rapid and the delayed effects could potentially be mediated by different processes. No evidence to support the transcriptional regulation of AMPAR subunits by glucocorticoids has been observed in in vitro studies [40] and, therefore, changes in proteins involved in regulating AMPAR delivery and/or membrane anchoring are hypothesized to be intermediate targets of increased corticosterone. Recently, the induction of the transcription factor serum- and glucocorticoid-inducible kinase (SGK) and the activation of the hydrolase Rab4 (a member of the Rab family of proteins involved in membrane trafficking events) were implicated in the delayed increase in surface expression of AMPARs and NMDARs in the PFC response to stress and corticosterone treatments $[61,62]$ and in the facilitation of working memory discussed above [57].

\section{Glucocorticoids can facilitate memory consolidation by interacting with synaptic NMDARs and their signaling cascades}

Classically, memory consolidation is believed to require changes in gene transcription and de novo protein synthesis [63] triggered by learning-activated molecular signaling cascades which include NMDAR activation and the subsequent activation of protein kinases, followed by activation of cAMP response element binding protein (CREB) [64,65]. More recently, epigenetic mechanisms - that are crucially involved in the regulation of gene transcription - have also been implicated in memory formation and maintenance [66]. Importantly, the activation of NMDARs, in addition to being required for many forms of synaptic plasticity and learning (Box 2) [67,68], has also been implicated in the effects of stress and glucocorticoids on hippocampal LTP and LTD $[37,69]$ and in some forms of learning. For example, amygdaloid NMDARs (that play a crucial role in fear extinction [70]) have also been implicated in the facilitating effect of glucocorticoids on fear extinction [71,72]. The interaction of glucocorticoids with NMDAR pathways is proposed to affect memory via changes in gene transcription (Figure 1e).

The involvement of epigenetic mechanisms in glucocorticoid-NMDAR interactions in the modulation of memory has been demonstrated recently. Specifically, evidence has been presented that glucocorticoid-induced enhancement of memory consolidation (as assessed in a forced swim test) took place through the activation of NMDARs and the downstream mitogen-activated protein kinase (MAPK) pathway, including activation of ERK (extracellular signal-regulated kinase)/MSK (mitogen and stress-activated protein kinase) and transcription factor Elk-1 in the dentate gyrus of the hippocampus [73-75]. Activation of this pathway was demonstrated to result in epigenetic changes including enhanced histone acetylation [73-75]. These epigenetic mechanisms were only efficient in the presence of activated GRs and they acted in synergy with NMDAR activation [74] (Figure 1e). Similar signaling and epigenetic pathways have been implicated in memory formation in other behavioral paradigms, including the water maze and fear conditioning [76]. Furthermore, epigenetic mechanisms were also recently described to be involved in corticosterone-induced (membrane GR-mediated) facilitation of long-term memory for object recognition (in the insular cortex) and object location (in the hippocampus), although the involvement of glutamatergic mechanisms was not explored in this study [77].

Although epigenetic mechanisms were not explored, the MAPK and $\mathrm{Ca}^{2+} /$ calmodulin-dependent protein kinase II (CaMKII) pathways have also been implicated, in parallel with rapid and delayed increases in the expression of glucocorticoid receptors (MR and GR, respectively), in the facilitating effects of stress on the long-term establishment of LTP in the hippocampus ([78]; but note that the same pathway was implicated in the detrimental effects of glucocorticoids on LTP in another study [79]). This facilitation was induced by a brief exposure to swim stress that transformed an electrically induced, protein-synthesis-independent early LTP (lasting for a maximum of $\sim 4-5 \mathrm{~h}$ ) into longer-lasting and protein-synthesis-dependent late LTP in the dentate gyrus [79].

\section{Glucocorticoids can facilitate memory consolidation through changes in gene transcription leading to increased glutamate release probability}

The identification of the key effector molecules that are regulated by glucocorticoid genomic actions and which affect memory consolidation was an open question for

learning. Subsequently, this mechanism increases the probability of activating postsynaptic glutamate receptors, at first predominantly AMPARs, thereby facilitating memory processes. (c) Glucocorticoids increase extracellular glutamate levels and activate extrasynaptic GluN2B-containing NMDARs. This pathway represents situations involving high to very high glucocorticoid levels that are not associated with relevant information processing in the circuit. High glutamate levels - accumulated extracellularly by glucocorticoid-mediated blockade of glutamate transporter activity - spill over and activate extrasynaptic GluN2B-containing NMDARs; this leads to endocytosis of GluA2-containing AMPARs. This mechanism is proposed to explain the facilitating effects of glucocorticoids on LTD and their impairing effects on LTP and cognitive processing. (d) Glucocorticoids increase excitatory transmission by inducing AMPAR synaptic delivery. Enhanced glucocorticoid levels can enhance AMPAR insertion at synaptic sites through both nongenomic (i.e. rapid) and genomic (i.e. delayed) effects. Such a mechanism helps to increase synaptic efficacy and learning and memory processes. (e) Glucocorticoids can lead to the activation of NMDARs and associated signaling cascades, resulting in epigenomic modifications. Glucocorticoid effects - via GRs - can trigger NMDAR-dependent cascades that are known to be implicated in memory consolidation, including activation of the MAPK and CaMKII pathways, and their subsequent phosphorylation and activation of transcription factors (such as CREB and Elk-1). This in turn leads to the modulation of epigenomic mechanisms, particularly chromatin modifications, which affect gene transcription. This mechanism can explain facilitatory effects of glucocorticoids on memory consolidation [83]. (f) Glucocorticoids can activate transcription factors and signaling cascades, leading to a delayed increase in the probability of glutamate release. In this case, glucocorticoid GR-mediated genomic actions are presynaptic and mediated by activation of the MAPK signaling pathway and the immediate early gene Erg-1. As a consequence, synapsin expression is increased and its phosphorylation is subsequently enhanced; this can trigger increased vesicular glutamate availability and release. This pathway can explain the facilitating effects of glucocorticoids on memory processes [80] - but note that the delayed timing of the enhanced probability of glutamate release (as opposed to the rapid release represented in panels B and C) affects late information processing rather than the earlier phases of learning. 
many years. However, two studies in recent years have identified a signaling cascade that mediates the enhancement of fear memories in mice elicited by activation of hippocampal GRs, with increased glutamate release being proposed as the ultimate molecular effector $[14,80]$. This cascade, which is required for glucocorticoid facilitation of memory, begins with increased expression of the transcription factor Egr-1 (early growth-response factor) by GR, followed by an increase in the expression and activity of the MAPK pathway. Activation of the MAPK pathway further enhances the expression of Egr1 and activates synapsin-Ia/Ib (a presynaptic vesicle-associated phosphoprotein). Changes in synapsin expression and activation would thus likely result in increased probability of glutamate release, hypothetically facilitating information processing and memory (Figure 1f). Note that this mechanism is distinct from and has a different temporal pattern (ie. delayed in time of onset) than the non-genomic, rapid and transient effects of glucocorticoids on glutamate release discussed before (Figure 1b).

\section{General principles for the diversity of glucocorticoid outcomes on memory processes and their link with glutamatergic pathways}

The literature linking glucocorticoid actions with glutamatergic mechanisms reveals a number of operating rules for the conditions in which glucocorticoids exert a diversity of effects in cognitive function. These general principles are outlined in the following sections.

(i) Facilitating effects on memory processes are observed when moderate-to-high glucocorticoid elevations converge in time with information processing

Memory processes are facilitated by glucocorticoid elevation (triggered by the task or induced by exogenous administration) that takes place over a time-period extending from shortly before training (i.e. less than $5 \mathrm{~min}$ before) to up to $1 \mathrm{~h}$ after training $[39,40,42-44,50]$. These circumstances foster both rapid and delayed (protein-synthesisdependent) synaptic delivery of AMPAR and/or NMDAR subunits [39,40,42,51]. A subsequent and/or additional pathway involves NMDAR-triggering of signaling cascades leading to (epi)genomic changes in gene transcription $[14,73-75,80]$, with at least one of these targets leading to increased probability of glutamate release [80]. In general, rapid effects are mediated by membrane-bound MRs or GRs, whereas delayed genomic effects are mediated by nuclear-localized GRs. These mechanisms account for the facilitating effects of glucocorticoids on the acquisition (presumably those related to rapid changes in glutamate receptor trafficking) and consolidation (presumably those resulting in changes in gene expression) of information.

However, there are exceptions to this timing constraint because some studies have reported that stress can facilitate memory processes despite lack of convergence between the time of glucocorticoid elevation and the time at which the cognitive challenge is given (with time-gaps of $2-4$ h) $[57,81]$ and at which changes in synaptic expression of glutamate receptors are observed [57]. A possible explanation for these divergent findings is that stress and glucocorticoids might facilitate certain cognitive processes by priming neural circuits relevant for subsequent task performance by stress. This possibility has been suggested in a human study in which stress exposure $2 \mathrm{~h}$ before learning specifically enhanced recall of stressor-related highly arousing words [81].

(ii) Detrimental effects on memory processes are observed when high-to-very high glucocorticoid elevations occur in an uncoupled manner during a time-window preceding cognitive challenge

This situation relates to glucocorticoid elevations elicited by stress or exogenous steroid administration given before the cognitive task (10-60 minutes before, but not at shorter time points) $[31,34,38]$. These conditions were shown to involve hippocampal GRs [20] and to lead to activation of extrasynaptic GluN2B-containing NMDARs and the endocytosis of GluA2-containing AMPARs [34,35]. Although these mechanisms were predominantly related to the detrimental effects of stress and glucocorticoids on retrieval $[34,38]$, note that under similar uncoupled conditions pre-training stress or glucocorticoid treatments can also impair memory formation [5].

(iii) Different cognitive outcomes are observed when glucocorticoid-induced increases in extracellular glutamate levels are mediated by actions on release versus uptake mechanisms

When corticosterone-induced extracellular glutamate increase takes place through an increase in release probability, positive effects on plasticity and information processing follow (probably because an increase in glutamate release probability would secure timing convergence between activity-induced glutamate release and its subsequent impact upon the activation of postsynaptic pathways) $[21,22,82]$. By contrast, if glutamate increase results from blockade of uptake mechanisms, negative effects are found $[20,34]$, with glutamate spillover reaching extrasynaptic GluN2B-containing NMDARs as the proposed mediating mechanism $[35,36]$.

(iv) Facilitating effects of glucocorticoids are observed for memory consolidation, whereas detrimental effects are observed during information retrieval

This general principle $[8,12]$ was always observed in the reviewed studies focusing on glucocorticoid-glutamatergic interactions - in other words, the positive actions of glucocorticoids on cognition were generally related to memory consolidation $[14,42,50,80,83]$ whereas impairing effects were linked to retrieval [34,38]). A key question to ask is whether these differential effects are due to a putative 'vulnerability' of these different cognitive processes [in either a positive (consolidation) or a negative (retrieval) manner] to stress or elevated glucocorticoids, or whether they are the consequence of serendipitous application of experimental designs in the respective studies with glucocorticoid timing and dosage (see above) being instead the key factors underlying the reported effects. The evidence supports the former possibility because the same glucocorticoid treatment can simultaneously inhibit the immediate recall of information while facilitating consolidation mechanisms involved in long-term memory formation $[84,85]$. This has been specifically shown for the consolidation of extinction processes related to the information whose recall is inhibited [85-87]. 
In the PFC, these dual glucocorticoid effects were shown to be mediated by a common neural mechanism [88]. However, note that although substantial evidence suggests that retrieval processes are particularly vulnerable to disruption by high to very high and uncoupled glucocorticoid levels, impaired learning and/or memory consolidation is also observed when a brief/mild learning experience such as object recognition [89] or contextual fear memory [11] - is presented during the time window of vulnerability after high to very high stress. The same type of treatments did not impair learning and memory when given before strong and/or extensive training [5,31]. These studies suggest that the nature of the cognitive challenge - through the characteristics of its associated neurocircuit recruitment - is a key determinant of vulnerability, with cognitive processes involving short (as frequently is the case for the recall tests) and/or mild challenges being particularly susceptible to disruption. Thus, recruitment of relevant cognitive networks might be particularly challenging and sensitive to interference when they are undergoing modifications in their activation threshold (e.g. those brought about through glucocorticoid-induced and GR-mediated LTD-like mechanisms). Delayed GR-mediated actions [4] involving changes in the levels of synaptic AMPARs [39] have been proposed to suppress efficient information processing by elevating the signal-to-noise ratio for new synaptic inputs coming to the network $[4,41]$.

\section{Towards a mechanistic model of glucocorticoid- glutamatergic interactions on memory processes}

Based on the reviewed literature, I propose the following two-component model whereby glucocorticoid effects on glutamatergic pathways could help to explain glucocorticoid actions on cognition. The model incorporates principles related to glucocorticoid 'timing' (with regards to the cognitive challenge) and 'dosage' (see Box 2) as well as to the characteristics of the neural recruitment triggered by the cognitive challenge. In addition, it emphasizes the relevance of the coupling between glucocorticoid elevation and neural activity related to information processing for the cognitive outcome.

The first prediction of the model is that positive effects of glucocorticoids will be found when there is a coupling between neural activity and moderate-to-high glucocorticoid-induced enhanced glutamate levels (i.e. from $5 \mathrm{~min}$ before to up to $1 \mathrm{~h}$ after information processing; Figure 1b) and/or AMPAR synaptic delivery and activation of associated signaling pathways (Figure 1d). Moderate-to-high glucocorticoid doses will increase glutamate levels by acting presynaptically on release probability (Figure 1b). This accounts both for those situations in which glucocorticoids triggered by (stressful) training contribute to the formation of a long-term memory (i.e. intrinsic stress; in which the timing is guaranteed by the nature of the physiological reaction to the event) and for those situations in which stress or glucocorticoids are administered either shortly before (i.e. extrinsic stress; in which case their impact upon glutamatergic pathways overlaps with the start of information processing) or after training (where glucocorticoids can contribute to memory consolidation by enhancing glutamatergic mechanisms that are naturally involved in these processes; Figure 1e,f). Therefore, this model regards consolidation processes as particularly suitable for facilitatation by glucocorticoid coupling with the learning event.

The second prediction of this model is that glucocorticoids will negatively affect cognition when the enhanced extracellular glutamate levels induced by exposure to high-to-very high corticosterone do not overlap with the relevant neural activity, but instead occur during an adjacent time interval (e.g. starting $\sim 10-60 \mathrm{~min}$ before). High-to-very high glucocorticoid doses will block glutamate reuptake, resulting in glutamate spillover that can then activate extrasynaptic GluN2B-containing NMDARs, resulting in endocytosis of GluA2-containing AMPARs (Figure 1c). This will increase the signal-to-noise ratio for new synaptic activity at the same synapse, increasing the demand for effective neural recruitment, and thereby increasing vulnerability to cognitive deficits. The degree of neural recruitment engaged by the cognitive task is, therefore, a defining factor for these delayed detrimental effects, with both retrieval processes and learning processes based on weak training experiences being particularly vulnerable to disruption.

Although this model accounts for multiple and opposing actions of glucocorticoids on cognition taking place in different brain regions, it does not directly address the role played by glucocorticoid-glutamatergic interactions involving the interplay between different brain regions (models involving dynamics of brain interactions are given in $[12,90,91])$, nor the relevance of other neurotransmitters and peptides [92] that can influence and modulate the aforementioned effects.

\section{Concluding remarks}

Here I have presented evidence that glucocorticoids act at several different levels within glutamatergic pathways, and that such effects underlie glucocorticoid-mediated effects on cognition. Although the different pathways have been presented independently (Figure 1), they should be considered as 'snapshots' of a more global picture that probably includes the sequential involvement of several (or all) of them. A key question for future studies will be to ascertain to what extent the enhancement of glutamatergic signaling (Figure 1b) is a necessary and/or sufficient requirement for triggering downstream signaling pathways (Figure $1 \mathrm{~d}-\mathrm{f}$ ) that ultimately result in long-lasting changes in synaptic efficacy and alterations in memory performance.

On the basis of the evidence reviewed I have proposed a two-component model aiming to explain glucocorticoid actions on cognition based on the specific effects of these hormones on glutamatergic pathways. The model foresees that positive effects of glucocorticoids will occur when there is a coupling between neural activity related to information processing in relevant circuits and moderate to high glucocorticoid-induced enhanced glutamate levels and/or AMPAR synaptic delivery. Conversely, negative effects will take place when high to very high corticosteroneinduced high extracellular glutamate levels are uncoupled, but closely linked in time to neural activity. Because glutamatergic mechanisms are central to synaptic plasticity and memory formation, the modulation of these pathways by glucocorticoids provides these hormones with a 
mechanism whereby they can directly affect cognitive functions, adding a new dimension to former views of glucocorticoid actions.

\section{Acknowledgments}

Work in the author's laboratory that relates to this topic was supported by grants from the European Union 7th Framework Program (MemStick), the Swiss Society for Neuroscience (grants 310000-120791; Sinergia CRSIK0-122697 and CRSIK0-122691) and by the Swiss Federal Institute for Technology. The author would like to thank previous and current coworkers, particularly L. Conboy and C. Venero, for their original contributions.

\section{References}

1 de Kloet, E.R. et al. (2008) Corticosteroid hormones in the central stress response: quick-and-slow. Front. Neuroendocrinol. 29, 268-272

2 McEwen, B.S. (1999) Stress and hippocampal plasticity. Annu. Rev. Neurosci. 22, 105-122

3 Sandi, C. (2004) Stress, cognitive impairment and cell adhesion molecules. Nat. Rev. Neurosci. 5, 917-930

4 Joëls, M. et al. (2006) Learning under stress: how does it work? Trends Cogn. Sci. 10, 152-158

5 Sandi, C. and Pinelo-Nava, M.T. (2007) Stress and memory: behavioral effects and neurobiological mechanisms. Neural Plast. 2007, 78970

6 Sandi, C. (1998) The role and mechanisms of action of glucocorticoid involvement in memory storage. Neural Plast. 6, 41-52

7 de Kloet, E.R. et al. (1999) Stress and cognition: are corticosteroids good or bad guys? Trends Neurosci. 22, 422-426

8 Roozendaal, B. (2002) Stress and memory: opposing effects of glucocorticoids on memory consolidation and memory retrieval. Neurobiol. Learn. Mem. 78, 578-595

9 Conrad, C.D. (2005) The relationship between acute glucocorticoid levels and hippocampal function depends upon task aversiveness and memory processing state. Nonlinearity Biol. Toxicol. Med. 3, 57-78

10 Joëls, M. (2006) Corticosteroid effects in the brain: U-shape it. Trends Pharmacol. Sci. 27, 244-250

11 Diamond, D.M. et al. (2007) The temporal dynamics model of emotional memory processing: a synthesis on the neurobiological basis of stress-induced amnesia, flashbulb and traumatic memories, and the Yerkes-Dodson law. Neural Plast. 2007, 60803

12 de Quervain, D.J. et al. (2009) Glucocorticoids and the regulation of memory in health and disease. Front. Neuroendocrinol. 30, 358-370

13 Schaaf, M.J. et al. (1999) Corticosterone effects on BDNF mRNA expression in the rat hippocampus during morris water maze training. Stress 3, 173-183

14 Revest, J.M. et al. (2005) The MAPK pathway and Egr-1 mediate stress-related behavioral effects of glucocorticoids. Nat. Neurosci. 8 , 664-672

15 Bisaz, R. et al. (2009) Learning under stress: a role for the neural cell adhesion molecule NCAM. Neurobiol. Learn. Mem. 91, 333-342

16 Bisaz, R. and Sandi, C. (2010) The role of NCAM in auditory fear conditioning and its modulation by stress: a focus on the amygdala. Genes Brain Behav. 9, 353-364

17 Prager, E.M. and Johnson, L.R. (2009) Stress at the synapse: signal transduction mechanisms of adrenal steroids at neuronal membranes. Sci. Signal. 2, re5

18 Venero, C. and Borrell, J. (1999) Rapid glucocorticoid effects on excitatory amino acid levels in the hippocampus: a microdialysis study in freely moving rats. Eur. J. Neurosci. 11, 2465-2473

19 Virgin, C.E., Jr et al. (1991) Glucocorticoids inhibit glucose transport and glutamate uptake in hippocampal astrocytes: implications for glucocorticoid neurotoxicity. J. Neurochem. 57, 1422-1428

20 Yang, C.H. et al. (2005) Behavioral stress enhances hippocampal CA1 long-term depression through the blockade of the glutamate uptake. J. Neurosci. 25, 4288-4293

21 Karst, H. et al. (2005) Mineralocorticoid receptors are indispensable for nongenomic modulation of hippocampal glutamate transmission by corticosterone. Proc. Natl. Acad. Sci. U.S.A. 102, 19204-19207

22 Olijslagers, J.E. et al. (2008) Rapid changes in hippocampal CA1 pyramidal cell function via pre- as well as postsynaptic membrane mineralocorticoid receptors. Eur. J. Neurosci. 27, 2542-2550
23 Wang, C.C. and Wang, S.J. (2009) Modulation of presynaptic glucocorticoid receptors on glutamate release from rat hippocampal nerve terminals. Synapse 63, 745-751

24 Musazzi, L. et al. (2010) Acute stress increases depolarization-evoked glutamate release in the rat prefrontal/frontal cortex: the dampening action of antidepressants. PLoS ONE 5, e8566

25 Tasker, J.G. et al. (2006) Minireview: rapid glucocorticoid signaling via membrane-associated receptors. Endocrinology 147, 5549-5556

26 Karst, H. et al. (2010) Metaplasticity of amygdalar responses to the stress hormone corticosterone. Proc. Natl. Acad. Sci. U.S.A. 107, 14449-14454

27 Duvarci, S. and Paré, D. (2007) Glucocorticoids enhance the excitability of principal basolateral amygdala neurons. J. Neurosci. $27,4482-4491$

28 Liebmann, L. et al. (2008) Differential effects of corticosterone on the slow afterhyperpolarization in the basolateral amygdala and CA1 region: possible role of calcium channel subunits. J. Neurophysiol. 99, 958-968

29 Sandi, C. et al. (1996) Novelty-related rapid locomotor effects of corticosterone in rats. Eur. J. Neurosci. 8, 794-800

30 Sandi, C. et al. (1996) Nitric oxide synthesis inhibitors prevent rapid behavioral effects of corticosterone in rats. Neuroendocrinology 63 446-453

31 de Quervain, D.J. et al. (1998) Stress and glucocorticoids impair retrieval of long-term spatial memory. Nature 394, 787-790

32 Diamond, D.M. et al. (1999) Exposing rats to a predator impairs spatial working memory in the radial arm water maze. Hippocampus 9, 542-552

33 Diamond, D.M. et al. (2006) Influence of predator stress on the consolidation versus retrieval of long-term spatial memory and hippocampal spinogenesis. Hippocampus 16, 571-576

34 Wong, T.P. et al. (2007) Hippocampal long-term depression mediates acute stress-induced spatial memory retrieval impairment. Proc. Natl. Acad. Sci. U.S.A. 104, 11471-11476

35 Fox, C.J. et al. (2007) Tyrosine phosphorylation of the GluA2 subunit is required for long-term depression of synaptic efficacy in young animals in vivo. Hippocampus 17, 600-605

36 Massey, P.V. et al. (2004) Differential roles of NR2A and NR2Bcontaining NMDA receptors in cortical long-term potentiation and long-term depression. J. Neurosci. 24, 7821-7828

37 Wiegert, O. et al. (2005) Glucocorticoid receptor activation selectively hampers N-methyl-D-aspartate receptor dependent hippocampal synaptic plasticity in vitro. Neuroscience $135,403-411$

38 Howland, J.G. and Cazakoff, B.N. (2010) Effects of acute stress and GluN2B-containing NMDA receptor antagonism on object and objectplace recognition memory. Neurobiol. Learn. Mem. 93, 261-267

39 Groc, L. et al. (2008) The stress hormone corticosterone conditions AMPAR surface trafficking and synaptic potentiation. Nat. Neurosci. $11,868-870$

40 Martin, S. et al. (2009) Corticosterone alters AMPAR mobility and facilitates bidirectional synaptic plasticity. PLOS ONE 4, e4714

41 Krugers, H.J. et al. (2010) Stress hormones and AMPA receptor trafficking in synaptic plasticity and memory. Nat. Rev. Neurosci. $11,675-681$

42 Conboy, L. and Sandi, C. (2010) Stress at learning facilitates memory formation by regulating AMPA receptor trafficking through a glucocorticoid action. Neuropsychopharmacology 35, 674-685

43 Sandi, C. et al. (1997) Experience-dependent facilitating effect of corticosterone on spatial memory formation in the water maze. Eur. J. Neurosci. 9, 637-642

44 Akirav, I. et al. (2004) A facilitative role for corticosterone in the acquisition of a spatial task under moderate stress. Learn. Mem. 11, 188-195

45 Saglietti, L. et al. (2007) Extracellular interactions between GluA2 and N-cadherin in spine regulation. Neuron 54, 461-477

46 Merino, J.J. et al. (2000) Regulation of hippocampal cell adhesion molecules NCAM and L1 by contextual fear conditioning is dependent upon time and stressor intensity. Eur. J. Neurosci. 12, 3283-3290

47 Venero, C. et al. (2006) Hippocampal up-regulation of NCAM expression and polysialylation plays a key role on spatial memory. Eur. J. Neurosci. 23, 1585-1595

48 Lopez-Fernandez, M.A. et al. (2007) Upregulation of polysialylated neural cell adhesion molecule in the dorsal hippocampus after 
contextual fear conditioning is involved in long-term memory formation. J. Neurosci. 27, 4552-4561

49 Sandi, C. and Rose, S.P. (1994) Corticosteroid receptor antagonists are amnestic for passive avoidance learning in day-old chicks. Eur. J. Neurosci. 6, 1292-1297

50 Sandi, C. and Rose, S.P. (1997) Training-dependent biphasic effects of corticosterone in memory formation for a passive avoidance task in chicks. Psychopharmacology 133, 152-160

51 Venero, C. and Sandi, C. (1997) Effects of NMDA and AMPA receptor antagonists on corticosterone facilitation of long-term memory in the chick. Eur. J. Neurosci. 9, 1923-1928

52 Roozendaal, B. and McGaugh, J.L. (1997) Basolateral amygdala lesions block the memory-enhancing effect of glucocorticoid administration in the dorsal hippocampus of rats. Eur. J. Neurosci. 9, 76-83

53 Whitlock, J.R. et al. (2006) Learning induces long-term potentiation in the hippocampus. Science 313, 1093-1097

54 Donley, M.P. et al. (2005) Glucocorticoid receptor antagonism in the basolateral amygdala and ventral hippocampus interferes with longterm memory of contextual fear. Behav. Brain Res. 164, 197-205

55 Rumpel, S. et al. (2005) Postsynaptic receptor trafficking underlying a form of associative learning. Science 308, 83-88

56 Zhou, M. et al. (2009) Fear conditioning enhances spontaneous AMPA receptor-mediated synaptic transmission in mouse hippocampal CA1 area. Eur. J. Neurosci. 30, 1559-1564

57 Yuen, E.Y. et al. (2009) Acute stress enhances glutamatergic transmission in prefrontal cortex and facilitates working memory. Proc. Natl. Acad. Sci. U.S.A. 106, 14075-14079

58 Schmidt, M. et al. (2010) Individual stress vulnerability is predicted by short-term memory and AMPA receptor subunit ratio in the hippocampus. J. Neurosci. 30, 16949-16958

59 Roozendaal, B. et al. (2004) The basolateral amygdala interacts with the medial prefrontal cortex in regulating glucocorticoid effects on working memory impairment. J. Neurosci. 24, 1385-1392

60 Luethi, M. et al. (2008) Stress effects on working memory, explicit memory, and implicit memory for neutral and emotional stimuli in healthy men. Front. Behav. Neurosci. DOI: 10.3389/ neuro.08.005.2008

$61 \mathrm{Liu}, \mathrm{W}$. et al. (2010) The stress hormone corticosterone increases synaptic alpha-amino-3-hydroxy-5-methyl-4-isoxazolepropionic acid (AMPA) receptors via serum- and glucocorticoid-inducible kinase (SGK) regulation of the GDI-Rab4 complex. J. Biol. Chem. 285, 6101-6108

62 Yuen, E.Y. et al. (2010) Mechanisms for acute stress-induced enhancement of glutamatergic transmission and working memory. Mol. Psychiatry DOI: $10.1038 / \mathrm{mp} .2010 .50$

63 Davis, H.P. and Squire, L.R. (1984) Protein synthesis and memory: a review. Psychol. Bull. 96, 518-559

64 Wang, H. et al. (2006) Molecular and systems mechanisms of memory consolidation and storage. Prog. Neurobiol. 79, 123-135

65 Bibb, J.A. et al. (2010) Cognition enhancement strategies. J. Neurosci. 30, 14987-14992

66 Day, J.J. and Sweatt, J.D. (2011) Cognitive neuroepigenetics: a role for epigenetic mechanisms in learning and memory. Neurobiol. Learn. Mem. DOI: 10.1016/j.nlm.2010.12.008

67 Bannerman, D.M. et al. (1995) Distinct components of spatial learning revealed by prior training and NMDA receptor blockade. Nature 378, 182-186

68 Morris, R.G. (2006) Elements of a neurobiological theory of hippocampal function: the role of synaptic plasticity, synaptic tagging and schemas. Eur. J. Neurosci. 23, 2829-2846

$69 \mathrm{Kim}$, J.J. et al. (1996) Behavioral stress modifies hippocampal plasticity through N-methyl-D-aspartate receptor activation. Proc. Natl. Acad. Sci. U.S.A. 93, 4750-4753

70 Walker, D.L. et al. (2002) Facilitation of conditioned fear extinction by systemic administration or intra-amygdala infusions of D-cycloserine as assessed with fear-potentiated startle in rats. J. Neurosci. 22, 2343-2351

71 Yang, Y.L. et al. (2006) Systemic and intra-amygdala administration of glucocorticoid agonist and antagonist modulate extinction of conditioned fear. Neuropsychopharmacology 31, 912-924

72 Yang, Y.L. et al. (2007) Glutamate NMDA receptors within the amygdala participate in the modulatory effect of glucocorticoids on extinction of conditioned fear in rats. Neuropsychopharmacology 32 1042-1051

73 Bilang-Bleuel, A. et al. (2005) Psychological stress increases histone H3 phosphorylation in adult dentate gyrus granule neurons: involvement in a glucocorticoid receptor-dependent behavioural response. Eur. J. Neurosci. 22, 1691-1700

74 Chandramohan, Y. et al. (2007) Novelty stress induces phosphoacetylation of histone $\mathrm{H} 3$ in rat dentate gyrus granule neurons through coincident signalling via the N-methyl-D-aspartate receptor and the glucocorticoid receptor: relevance for c-fos induction. J. Neurochem. 101, 815-828

75 Chandramohan, Y. et al. (2008) The forced swimming-induced behavioural immobility response involves histone H3 phosphoacetylation and c-Fos induction in dentate gyrus granule neurons via activation of the $\mathrm{N}$-methyl-D-aspartate/extracellular signalregulated kinase/mitogen- and stress-activated kinase signalling pathway. Eur. J. Neurosci. 27, 2701-2713

76 Chwang, W.B. et al. (2007) The nuclear kinase mitogen- and stressactivated protein kinase 1 regulates hippocampal chromatin remodeling in memory formation. J. Neurosci. 27, 12732-12742

77 Roozendaal, B. et al. (2010) Membrane-associated glucocorticoid activity is necessary for modulation of long-term memory via chromatin modification. J. Neurosci. 30, 5037-5046

78 Ahmed, T. et al. (2006) Long-term effects of brief acute stress on cellular signaling and hippocampal LTP. J. Neurosci. 26, 3951-3958

79 Yang, C.H. et al. (2004) Behavioral stress modifies hippocampal synaptic plasticity through corticosterone-induced sustained extracellular signal-regulated kinase/mitogen-activated protein kinase activation. J. Neurosci. 24, 11029-11034

80 Revest, J.M. et al. (2010) The enhancement of stress-related memory by glucocorticoids depends on synapsin-Ia/Ib. Mol. Psychiatry 1125 , $1140-1151$

81 Smeets, T. et al. (2009) Stress selectively and lastingly promotes learning of context-related high arousing information. Psychoneuroendocrinology 34, 1152-1161

82 Wiegert, 0. et al. (2006) Timing is essential for rapid effects of corticosterone on synaptic potentiation in the mouse hippocampus. Learn. Mem. 13, 110-113

83 Reul, J.M. et al. (2009) Epigenetic mechanisms in the dentate gyrus act as a molecular switch in hippocampus-associated memory formation. Epigenetics 4, 434-439

84 Okuda, S. et al. (2004) Glucocorticoid effects on object recognition memory require training-associated emotional arousal. Proc. Natl. Acad. Sci. U.S.A. 101, 853-858

85 Soravia, L.M. et al. (2006) Glucocorticoids reduce phobic fear in humans. Proc. Natl. Acad. Sci. U.S.A. 103, 5585-5590

86 Aerni, A. et al. (2004) Low-dose cortisol for symptoms of posttraumatic stress disorder. Am. J. Psychiatry 161, 1488-1490

87 Cai, W.H. et al. (2006) Postreactivation glucocorticoids impair recall of established fear memory. J. Neurosci. 26, 9560-9566

88 Barsegyan, A. et al. (2010) Glucocorticoids in the prefrontal cortex enhance memory consolidation and impair working memory by a common neural mechanism. Proc. Natl. Acad. Sci. U.S.A. 107, $16655-16660$

89 Baker, K.B. and Kim, J.J. (2002) Effects of stress and hippocampal NMDA receptor antagonism on recognition memory in rats. Learn. Mem. 9, 58-65

90 Roozendaal, B. et al. (2009) Glucocorticoid effects on memory consolidation depend on functional interactions between the medial prefrontal cortex and basolateral amygdala. J. Neurosci. 29, 1429914308

91 Roozendaal, B. et al. (2009) Stress, memory and the amygdala. Nat. Rev. Neurosci. 10, 423-433

92 Joëls, M. and Baram, T.Z. (2009) The neuro-symphony of stress. Nat. Rev. Neurosci. 10, 459-466

93 Joëls, M. et al. (2008) The coming out of the brain mineralocorticoid receptor. Trends Neurosci. 31, 1-7

94 Joëls, M. and Krugers, H.J. (2007) LTP after stress: up or down? Neural Plast. 2007, 93202

95 Salehi, B. et al. (2010) Learning under stress: The inverted-U-shape function revisited. Learn. Mem. 17, 522-530

96 Collingridge, G.L. et al. (2009) A nomenclature for ligand-gated ion channels. Neuropharmacology 56, 2-5 
97 Kessels, H.W. and Malinow, R. (2009) Synaptic AMPA receptor plasticity and behavior. Neuron $61,340-350$

$98 \mathrm{Liu}, \mathrm{Y}$. et al. (2010) A single fear-inducing stimulus induces a transcription-dependent switch in synaptic AMPAR phenotype. Nat. Neurosci. 13, 223-231
99 Rao, V.R. and Finkbeiner, S. (2007) NMDA and AMPA receptors: old channels, new tricks. Trends Neurosci. 30, 284-291

100 Yashiro, K. and Philpot, B.D. (2008) Regulation of NMDA receptor subunit expression and its implications for LTD LTP, and metaplasticity. Neuropharmacology 55, 1081-1094 